\title{
Assessment of drug prescribing pattern using WHO indicators in outpatient departments of a tertiary care teaching hospital in North India
}

\author{
Sanjay Khattri ${ }^{1}$, Sartaj Hussain ${ }^{1}$, Suraj S Yadav ${ }^{1}$, Manish Bajpai ${ }^{2}$, Kamal K Sawlani ${ }^{3}$ \\ ${ }^{\text {I}}$ Department of Pharmacology, King George's Medical University, U. P., Lucknow, India, ${ }^{2}$ Department of Physiology, \\ King George's Medical University, U. P., Lucknow, India, ${ }^{3}$ Department of Medicine, King George's Medical University, \\ U. P., Lucknow, India
}

Background: Irrational prescribing is prevalent worldwide but is a major problem in the developing countries. The World Health Organization (WHO) core indicators help to improve the prescribing patterns and thus promote the rational use of drugs in a health care facility.

Objectives: To assess the prescribing pattern of in outpatient department (OPD) of Medicine, Surgery and Pediatrics at a tertiary care teaching and research institute.

Methods: A cross sectional study was carried out over a period of one month. Four hundred prescriptions from each department were randomly collected and analyzed using WHO prescribing indicators.

Results: The average number of drugs per prescription in Medicine, Surgery and Pediatrics OPD was three, three and two, respectively. The drugs prescribed by generic name were only one percent in each department. The total percentage of encounters with antibiotics was highest in Surgery (45\%) followed by Medicine (40\%) and Pediatrics (34\%). The total percentage of encounters with injectables in Medicine, Surgery and Pediatrics OPD was $2 \%, 4 \%$ and $12 \%$, respectively. The percentage of drugs prescribed from the Essential Drug List (National) was highest in Pediatrics (22\%) followed by Medicine (19\%) and Surgery (15\%).

Conclusions: The prescribing practices were not appropriate as they consist of polypharmacy, lesser prescription by generic name, and over prescription of antibiotics. Recommendations to change the ongoing prescribing practices should be based on the Standard Treatment Guidelines, Essential Drug List and Antibiotic policy or by following the information, education, and communication (IEC) interventions. 